OPEN ACCESS

Edited by:

Leslie Yeo,

RMIT University, Australia

Reviewed by:

Miriam Mba

University of Padua, Italy

Omid Akhavan,

Sharif University of Technology, Iran

*Correspondence:

Alex Savchenko

asavtchenko@nanotoolsbio.com

Elena Molokanova

emolokanova@nanotoolsbio.com

Specialty section:

This article was submitted to

Nanobiotechnology,

a section of the journal

Frontiers in Bioengineering and

Biotechnology

Received: 18 October 2021 Accepted: 10 November 2021 Published: 07 December 2021

Citation:

Savchenko A, Yin RT, Kireev D,

Efimov IR and Molokanova E (2021)

Graphene-Based Scaffolds:

Fundamentals and Applications for

Cardiovascular Tissue Engineering.

Front. Bioeng. Biotechnol. 9:797340.

doi: $10.3389 /$ fbioe. 2021.797340

\section{Graphene-Based Scaffolds: Fundamentals and Applications for Cardiovascular Tissue Engineering}

\author{
Alex Savchenko ${ }^{1 *}$, Rose T. Yin ${ }^{2}$, Dmitry Kireev ${ }^{3}$, Igor R. Efimov ${ }^{2}$ and Elena Molokanova ${ }^{1,4 *}$ \\ ${ }^{1}$ Nanotools Bioscience, La Jolla, CA, United States, ${ }^{2}$ Department of Biomedical Engineering, The George Washington University, \\ Washington, DC, United States, ${ }^{3}$ Department of Electrical and Computer Engineering, Microelectronics Research Center, The \\ University of Texas at Austin, Austin, TX, United States, ${ }^{4}$ Neurano Bioscience, La Jolla, CA, United States
}

Cardiac tissue engineering requires materials that can faithfully recapitulate and support the native in vivo microenvironment while providing a seamless bioelectronic interface. Current limitations of cell scaffolds include the lack of electrical conductivity and suboptimal mechanical properties. Here we discuss how the incorporation of graphene into cellular scaffolds, either alone or in combination with other materials, can affect morphology, function, and maturation of cardiac cells. We conclude that graphene-based scaffolds hold great promise for cardiac tissue engineering.

Keywords: graphene, scaffold, tissue engineering, biocompability, cardiomyocite, cardiac

\section{INTRODUCTION}

Cardiomyocytes are electrically and mechanically active cells. Therefore, to efficiently support their activities, the cardiac substrates/scaffolds must have matching properties in order to faithfully recapitulate the functional behavior of the myocardium. Despite technological advances, this task remains very challenging, and new materials that can address these problems are in high demand.

Graphene, the newest member of the carbon allotrope family, is an exceptional candidate for this role. Although other members of this family are already famous [e.g., diamonds (discovered in the 4th century BC in India), graphite (discovered in the 16th century in England), and fullerenes (synthesized in 1985)], graphene could outshine them all. Graphene, a two-dimensional (2D) carbon crystal, is often described using superlatives: graphene is the thinnest $(0.335 \mathrm{~nm})$, the lightest $(0.77$ $\mathrm{mg} / \mathrm{m} 2$ ), and the strongest (42 N/m) material (Novoselov et al., 2004; Lee et al., 2008; Geim, 2009; Geim and Novoselov, 2007; Novoselov et al., 2012). Electrical, mechanical, magnetic, optical, and thermal properties of graphene are as just as exceptional, which makes graphene a material unsurpassed in its potential for bioelectronic interfaces and cellular scaffolds. As discussed in this mini-review, the integration of graphene into $2 \mathrm{D}$ cell substrates and threedimensional (3D) scaffolds (Figure 1) produces more physiological microenvironment with such features as electrical conductivity, nano-scale topography, stretchability, and flexibility.

\section{GRAPHENE AND ITS FUNDAMENTALS}

Graphene came into the spotlight in 2010 with the Nobel Prize in Physics awarded "for groundbreaking experiments regarding the two-dimensional material graphene" (Geim, 2009; Novoselov et al., 2012). It became clear that exceptional physicochemical properties of graphene materials create exciting opportunities for development of novel and efficient graphene-based bioengineering systems. 
In graphene, carbon atoms are positioned $0.142 \mathrm{~nm}$ apart in a hexagonal lattice. All atoms are $\mathrm{sp}^{2}$-hybridized, and each atom allocates 3 electrons from its 4 outer shell electrons to form equivalent covalent $\sigma$-bonds with its three neighboring atoms. The $4^{\text {th }}$ electron which occupies a pz orbital is forming a $\pi$ bond. Unlike the sp2 orbitals, the pz orbitals do not directly overlap, and these $4^{\text {th }}$ electrons ( $\pi$ electrons) operate in the $3^{\text {rd }}$ dimension (above and below a 2D graphene sheet), allowing them to be highly mobile, behave as massless particles, and experience ballistic transport without scattering (Novoselov et al., 2005; Zhang et al., 2005; Geim and Novoselov, 2007; Castro Neto et al., 2009; Tielrooij et al., 2013).

The band structure of pristine graphene consists of a filled valence band and an empty conduction band that cross at the Dirac points. Undoped graphene does not have a band gap and, therefore, is considered a zero-gap semiconductor, or semimetal
(Novoselov et al., 2005; Meric et al., 2008). Graphene has very low electrical resistivity, the intrinsic mobility of its electrons is very high (Bolotin et al., 2008) ( 200,000 $\mathrm{cm}^{2} /$ Vs vs. $\sim 1,400 \mathrm{~cm}^{2} / \mathrm{Vs}$ in silicon), and the graphene's current density is $\sim 1,000,000$ times greater than in copper (Chen et al., 2008).

Strong carbon-carbon in-plane $\sigma$-bonds (the bonding energy of $4.93 \mathrm{eV}$ ) (Brenner and Harrison, 2002) make graphene the strongest material in nature: e.g., the tensile strength of graphene is $130 \mathrm{GPa}$ compared to $0.4 \mathrm{GPa}$ for structural steel or $70 \mathrm{GPa}$ for Kevlar. Composite materials with graphene greatly benefited from its low density $\left(\sim 2300 \mathrm{~kg} / \mathrm{m}^{3}\right)$ and large Youngs modulus ( $\sim \mathrm{TPa})$ (Lee et al., 2008). Defect-free graphene sheets exhibit impressive elastic properties and can restore its initial size after strain. Being pliable, graphene can take any form desired. Graphene is also stretchable up to $20 \%$ of its initial length.

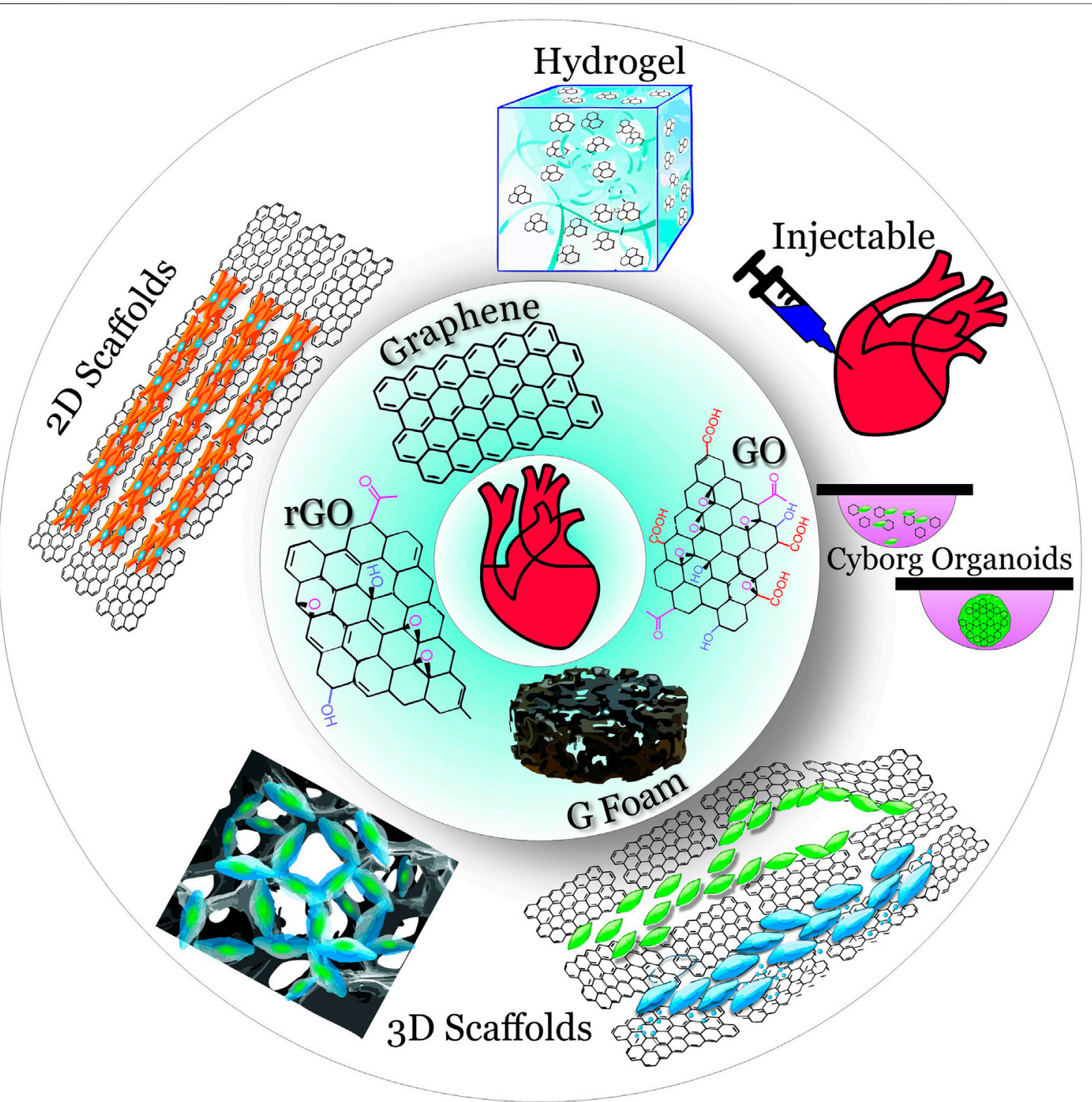

FIGURE 1 | Overview of graphene materials (inner circle) used in 2D and 3D scaffolds (outer circle) for cardiac tissue engineering. 
Graphene oxide (GO) is an oxidized form of graphene containing oxygen functional groups, the presence of which, among other consequences, leads to the disruption of the sp2 structure, increased interlayer spacing, and low electrical conductivity. Reduced graphene oxide ( $\mathrm{rGO}$ ) is a deoxygenized form of GO. Although the graphene structure in $\mathrm{rGO}$ is not completely restored by the reduction processes due to some remaining oxygen groups and surface defects, removal of oxygen groups and the increased $\mathrm{C} / \mathrm{O}$ ratio leads to restoration of the $\mathrm{sp} 2$ structure, increased mechanical strength, surface area and stability, hydrophobic properties, and the increase of electric conductivity up to $6300 \mathrm{~S} \mathrm{~cm}^{-1}$ and high mobility of $320 \mathrm{~cm}^{2} \mathrm{~V}^{-1} \mathrm{~s}^{-1}$ (Smith et al., 2019; Lesiak et al., 2021).

\section{BIOCOMPATIBILITY}

The utmost requirement for any biomedical material is biocompatibility. The very first biological studies of graphene sometimes produced contradictory and/or inconclusive results (Pinto et al., 2013) due to the novelty of graphene materials, the lack of the systematic classification (Wick et al., 2014; Park et al., 2017), and challenges associated with the interdisciplinary nature of nanobiotechnology (Reina et al., 2017). One of the striking examples of biocompatibility controversy surrounding graphene materials is their purported antibacterial properties. Initially, several studies claimed that all graphene materials exhibit antibacterial properties (Akhavan and Ghaderi, 2010; Hu et al., 2010). However, a comprehensive evaluation of graphene samples from different commercial and academic sources by a group from the University of Manchester that included Prof. Novoselov, the Nobel Prize laureate for graphene research, discovered that, in fact, the presence of such cytotoxic impurities as sulfur, boron, and sodium nitrate in graphene samples was the determinant factor for their ability to kill E. Coli (Barbolina et al., 2016).

Over subsequent years, it was established that a chemical synthesis route, purity, the number of layers, lateral dimensions, edge effects, defects, doping (intended and unintended), surface functionalization (intended and unintended), and dosage are the factors affecting how graphene may interact with cells (Pang et al., 2017; Seifi and Kamali, 2021). After becoming aware of the criticality of these parameters, scientists can now devise rational bioengineering approaches to synthesize cell-friendly graphene materials and to fabricate biocompatible graphene-based biointerfaces. For example, green-chemistry synthesis methods are highly advisable (Fernández-Merino et al., 2010; Khosroshahi et al., 2018; Regis et al., 2021), and graphene materials should be processed to eliminate various contaminants that might be cytotoxic on their own. Using large-surface grapheneintegrated substrates could eliminate such problems as potential physical damage from sharp edges of graphene sheets. Using large ( $>$ few microns) graphene flakes or combining of graphene with other materials (e.g., polymers, polysaccharides, hydrogels) can help to avoid endocytosis and potential generation of redox species occurring at the nanoscale.
Currently, the scientific community appears to be in agreement that properly engineered graphene materials are not only biocompatible but often superior in providing a microenvironment necessary for cell growth, differentiation, and development (Kim et al., 2013; Lee et al., 2014; Wang et al., 2017; Kenry et al., 2018).

To evaluate the graphene effects on cell stress, several studies monitored such highly sensitive cell viability indicators as autophagy levels and mitochondrial morphology and membrane potential (Rastogi et al., 2017; Rastogi et al., 2020; Lasocka et al., 2021). These studies determined that graphene has no detectable adverse effect on cell stress and suggested that graphene affects the cell-substrate interactions and promotes cell adhesion and cell proliferation through unique surface topography and adaptable surface chemistry.

Excellent biocompatibility of graphene was also demonstrated in several in vitro models of cardiac syncytium (e.g., neonatal rat ventricular cardiomyocytes, mouse embryonic stem cell (ESC)derived cardiomyocytes, and human induced pluripotent stem cell (hiPSC)-derived cardiomyocytes) cultured on graphenebased planar substrates fabricated from rGO or chemicalvapor-deposition (CVD) graphene. Further, zebrafish embryos injected with graphene flakes $(5-20 \mu \mathrm{m}$ in diameter) were exhibiting the basal heart rates similar to control embryos, and all but one zebrafish retained full viability 3 days later, which confirms biocompatibility of graphene in vivo (Savchenko et al., 2018).

\section{D SCAFFOLDS}

Graphene substrates are highly biocompatible with monolayer cardiomyocyte cultures (Kim et al., 2013) and appear to be a superior alternative to Matrigel, a substrate commonly used during cardiomyocyte differentiation from stem cells (Hitscherich et al., 2018). Incorporation of graphene into cell substrates was shown to markedly enhance the maturity and electrophysiological properties of hiPSC-cardiomyocytes that otherwise exhibit embryonic rather than adult-like phenotypes. Recent studies evaluating hiPSC-cardiomyocytes cultured on a single graphene layer demonstrated that these cells exhibit a more mature phenotype with improved myofibril alignment and density, increased $\mathrm{Cx} 43$ expression, and faster conduction velocity (from 2.2 to $5.3 \mathrm{~cm} / \mathrm{s}$ ). Calcium handling of hiPSCcardiomyocytes on graphene also became more mature: the expression levels of ryanodine receptors and sarcoendoplasmic reticulum $\mathrm{Ca}^{2+}$-ATPase were increased, and calcium transients exhibited a greater amplitude (Wang et al., 2017). This study suggested that a conductive surface of graphene mimicked the heart's microenvironment and facilitated its intrinsic electrical propagation properties to promote maturation of hiPSCcardiomyocytes.

Graphene can be combined with other materials to provide multifaceted advantages for hiPSC-cardiomyocytes. One such example is a hybrid collagen/graphene substrate, where collagen provides the biological support while graphene modulates the substrate stiffness and provides the electrical 
conductivity similar to conductivity of human cardiac tissues. When murine ESC-derived cardiomyocytes were cultured on such substrates (Ryan et al., 2018), they became more aligned and elongated with improved cross-striated sarcomeric structures, suggesting improved maturity.

Hybrid rGO-collagen substrates also produced significant improvements in mechanical and electrical properties of cardiomyocytes in 7 days and led to upregulation of cardiac gene expression involved in electrical coupling $(\mathrm{Cx} 43)$, muscle contraction and relaxation (troponin-T), and cytoskeleton alignment (actinin-4) even without electrical stimulation (Norahan et al., 2019).

Cardiomyocytes on a vitronectin-coated graphene substrate exhibited enhanced expression of cardiomyogenic markers and cardiac-specific extracellular matrix genes even without addition of cardiomyogenic factors. These results suggest that, by promoting the absorption and correct presentation of vitronectin, graphene induces mesodermal and endodermal lineage signaling which supports the cardiac development (Lee et al., 2014).

Another study transferred graphene films onto polymeric topographic substrates to create a conductive nanopatterned anisotropic scaffold. Cardiomyocytes on this scaffold demonstrated more mature structural and functional properties. These cardiomyocytes exhibited significantly increased z-band widths, greater sarcomere lengths, and increased expression of Cx43. Additionally, the amplitude of calcium transients and the action potential duration were significantly greater, suggesting enhanced maturation of cardiomyocytes (Smith et al., 2017).

\section{D SCAFFOLDS}

Since cardiac tissues in vivo are inherently $3 \mathrm{D}$, a similar geometric arrangement of engineered heart tissues (EHTs) would be more desirable to ensure faithful recapitulation of cell-to-cell coupling, and complex organization and function. Incorporation of graphene into cardiac 3D scaffolds can lead to anisotropic nanotopology, greatly enhanced electroconductive properties, and provide the ability to perform cell stimulation and monitoring via cell scaffolds.

A graphene foam (Hu et al., 2016; Krueger et al., 2016) itself can serve as a $3 \mathrm{D}$ sponge-like scaffold with interconnected pore structure, extended surface area, and nano-micro-scale topographical surface. This scaffold allows for more efficient cell-cell communication and transport of oxygen and nutrients than 2D substrates. Rat neonatal cardiomyocytes on a grapheneenhanced nickel foam were efficiently integrated into the scaffold with adequate biocompatibility and exhibited increased Cx43 gene expression even without direct electrical stimulation (Bahrami et al., 2019). An electrically active graphene foam was also developed to culture cardiomyocytes and directly monitor their extracellular action potentials at the same time (Ameri et al., 2016).

Another option is to directly embed graphene materials into $3 \mathrm{D}$ preparations. This approach was shown to increase the number of successfully differentiated 3D embryoid bodies and improve the mechanical and electrical properties of differentiated cardiomyocytes (Ahadian et al., 2016). Furthermore, when electrical stimulation was applied to these graphene-enhanced embryoid bodies, they exhibited more mature sarcomeric structures and became more physiologically active.

Park et al. combined GO flakes (1-6 $\mu \mathrm{m}$ in diameter) with MSCs and discovered that it led to more efficient engrafting of cells at the ischemic lesion site (Park et al., 2015). It appears that the addition of GO may combat the reactive oxygen species abundant in ischemia, prevent anoikis, and improve the therapeutic efficacy of MSCs implantation. This study also determined that paracrine secretion by engrafted cells successfully promoted angiogenesis, alleviated apoptosis in the infarcted region, and decreased infarction size.

Alternatively, cardiomyocytes and graphene materials can be combined to fabricate a complex multilayer cell construct. Shin et al. deposited a poly-L-lysine-GO nanofilm layer onto a homogenous layer of cardiomyocytes, seeded another layer of cells on top of the graphene-enhanced nanofilms, and then repeated these steps until the constructs comprised of 3 or more cell layers (Shin et al., 2014). This scaffold exhibited the elastic modulus of $\sim 10 \mathrm{kPa}$, resulted in increased expression of $\alpha$-actinin, and supported strong spontaneous synchronous contraction (20-30 BPM).

\section{ELECTROSPUN 3D SCAFFOLDS}

Graphene-containing 3D scaffolds can also be fabricated by electrospinning to develop the scaffolds with enhanced mechanical and electrical properties (Zhao et al., 2018; Chen et al., 2019; Ghasemi et al., 2019).

(Hitscherich et al., 2018) employed electrospinning to create a 3D nanofibrous graphene and poly (caprolactone) scaffold where graphene, distributed throughout the scaffold, enabled electrical stimulation of cells. This scaffold enhanced cell-cell coupling and improved the calcium handling in mouse ESC-derived cardiomyocytes as evidenced by increased Cx43 expression, improved cardiomyocyte organization and sarcomere alignment, and significantly increased calcium transient amplitudes and fractional release of calcium ions per beat.

Nazari et al. incorporated rGO-silver nanocomposites into polyurethane nanofibers using used electrospinning and demonstrated that human cardiac progenitor cells cultured on these scaffolds displayed great biocompatibility and cell attachment, and exhibited the upregulation of several cardiac-specific genes (e.g., GATA-4, Tbx18, troponin T, and $\alpha$-MHC) (Nazari et al., 2019).

In another development, poly (caprolactone) (PCL)/poly (glycerol sebacate) (PGS) nanofibers were fabricated via electrospinning, and graphene $(0.25,0.75$, or $1 \% \mathrm{wt})$ was added to PCL/PGS nanofibers. Addition of graphene to PCL/PGS nanofibrous scaffolds led to improved electrical conductivity, balanced hydrophilicity, and increased surface roughness. Adhesion, growth, migration, proliferation, and viability of cultured human cardiomyocytes were increased with the increase of the ratio of graphene in PCL/PGS scaffolds (Fakhrali et al., 2021). 
Talebi et al. used electrospinning technique to fabricate highly hydrophilic fibrous scaffolds composed of polycaprolactone/ chitosan/polypyrrole (PCP) and graphene (Talebi et al., 2020). PCP-graphene scaffolds were able to more closely mimic the elasticity and electrical conductivity of the native myocardial tissue, and to support biological and functional performance of murine ESC-derived cardiomyocytes.

To improve nanotopology and electrical conductivity of silk fibroin (SF) scaffolds, rGO nanosheets were incorporated into SF nanofibers via electrospinning. When used for cardiac differentiation of human iPSCs transfected with TBX18 gene, rGO-SF scaffolds exhibited improved mechanical and electrical properties, acceptable biocompatibility, considerable cell attachment, enhanced maturity and upregulation of cardiac genes (e.g., GATA-4, c-TnT, and $\alpha$-MHC) (Nazari et al., 2020). Alternatively, a layer of rGO can be deposited on the surface of electrospun SF substrates, also resulting in enhanced electrical conductivity of scaffolds and improved maturity of neonatal rat cardiomyocytes (Zhao et al., 2018).

\section{HYBRID HYDROGELS}

The incorporation of graphene materials into hydrogels results in hybrid electroactive scaffolds with improved cell adhesion, desirable mechanical properties, and low immunogenicity.

\section{In Vitro Applications}

Zhang et al. used a microcontact printing to create patterned genipin-cross-linked gelatin hydrogels containing GO. When cultured on this substrate, neonatal rat ventricular cardiomyocytes were aligned along the micropatterned scaffolds and demonstrated significant improvements in their structural and functional maturity: 1) more cardiomyocytes were binucleated and had longer sarcomere lengths much sooner; 2) the cardiac gene expression (e.g., Actn and $c T n T$ ) improved; 3) cardiomyocytes reached synchronized contractions within $48 \mathrm{~h}$, contracted in a more uniaxial manner and with increased amplitude, and continued contracting for up to 3 months (Zhang et al., 2019).

rGO can be incorporated into a gelatin methacryloyl (GelMA) hydrogel, which leads to significant enhancement of electrical and mechanical properties of the hybrid material (Shin et al., 2016). Neonatal rat cardiomyocytes on hybrid rGO-GelMA scaffolds exhibited uniformly distributed cell-cell junctions between neighboring cells, better defined and partially uniaxially aligned sarcomeric structures, improved cell viability, proliferation, maturation, stronger contractility, and faster spontaneous contraction rate compared cardiomyocytes to pristine GelMA hydrogels.

Another study utilized GelMA hydrogels with incorporated carbon nanotubes, GO, or rGO to compare the effects of these materials on the structural organization and functionality of hiPSC-cardiomyocytes (Lee et al., 2019). It was determined that electrically conductive rGO-GelMA scaffolds were more efficient in promoting mature morphology of cardiomyocytes, supporting their viability, and increasing the expression of functional cardiac markers than relatively non-conductive GO-GelMA scaffolds. Cardiomyocytes on rGO-GelMA scaffolds exhibited more mature rod-like morphology and higher expression levels of functional cardiac markers (e.g., Cx43 and troponin I), indicative of improved metabolic coupling and more mature excitation-contraction apparatus. Expression of mechanosensors (e.g., integrin, vinculin, and alpha-actinin) was also increased, leading to more robust contractions. Interestingly, cardiomyocytes on GO-GelMA scaffolds exhibited an atrial-like electrophysiological phenotype, while cardiomyocytes on rGO-GelMA scaffolds presented a mixed atrial/ventricular phenotype (Lee et al., 2019).

To engineer advanced EHTs, Tsui et al. used a decellularized extracellular matrix harvested from the left ventricular myocardium of porcine hearts to create a hydrogel composite scaffold with a preserved tissue-specific protein profile and a tunable stiffness enhanced by rGO (Tsui et al., 2021). Mechanical and electrical properties of these hydrogels were tuned by modulating rGO content and degree of reduction. Cardiac tissues engineered with this scaffold showed the increased expression levels of CX43, indicating improved cell-cell connectivity. Multiple functional improvements such as calcium handling, action potential duration, twitch forces, and conduction velocity were also induced by these hybrid rGOcontaining scaffolds.

Jing et al. developed polydopamine-based chitosan/GO composite hydrogels and demonstrated that addition of GO increased the hydrogel's adhesion strength by 300\%, and improved its electrical conductivity (Jing et al., 2017). Further, $\pi-\pi$ stacking, hydrogen bonding, and supramolecular interactions endowed chitosan/GO hydrogels with high stability, excellent mechanical properties, extended lifespan, self-healing properties, and fast-recovery ability. These hydrogels enhanced cell proliferation and viability and supported faster spontaneous beating rates in hESC-derived cardiomyocytes. These findings were confirmed in another study that tested porous chitosan/GO conductive scaffolds using cardiac H9C2 cell line and established that chitosan/GO scaffolds promoted cell attachment, viability, and upregulation of certain cardiac-specific genes (Jiang et al., 2019).

\section{In Vivo Applications}

Myocardial infarction causes irreversible damage to myocardium and results in an increased risk of heart failure and sudden cardiac death. One strategy to address this problem is to integrate new MSCs or cardiomyocytes into the remaining myocardium. Graphene-containing injectable hydrogels could improve the efficiency of engraftment and enhance the survival of implanted cells.

An injectable rGO-enhanced alginate hydrogel was used for culturing MSCs (Karimi Hajishoreh et al., 2020), resulting in the cell viability two-fold higher as compared to a pure alginate or a plastic substrate. Further, rat neonatal cardiac cells encapsulated in the alginate-rGO had significantly upregulated gene expression of $\operatorname{TrpT}-2, C x 43$, and Actn4 even without electrical stimulation. 
Another study combined methacryloyl-substituted tropoelastin with GO nanoparticles to engineer an injectable, stretchable, conductive hydrogel with an enhanced elasticity and toughness (Annabi et al., 2016). This GO-containing advanced hydrogel exhibited high biocompatibility, promoted the growth and proliferation of cells, and triggered minimal inflammatory response. Neonatal rat cardiomyocytes cultured on this hydrogel developed well-aligned sarcomeric structures that are similar to that of the native ventricular myocardium. Importantly, these cardiomyocytes could be depolarized at a lower excitation threshold, which points to their improved maturity.

To address the loss of vasculature at the ischemic site, Paul et al. employed an injectable graphene-enhanced hydrogel composed of a low-modulus methacrylated gelatin wtih polyethylenimine functionalized GO nanosheets to deliver a proangiogenic vascular endothelial growth factor plasmid DNA to the damaged myocardium (Paul et al., 2014). In a rat model of acute myocardial infarction, this hydrogel significantly decreased the scar area, alleviated inflammation at the infarction site, and improved systolic function as demonstrated by an improved ejection fraction.

Zhao et al. developed an injectable Reverse Thermal Gel (RTG) functionalized with GOs (GO-RTG) and capable of forming a $3 \mathrm{D}$ matrix at $37^{\circ} \mathrm{C}$ (Zhao, 2019). Using neonatal rat ventricular cardiomyocytes, this study demonstrated that these conductive 3D GO-RTG scaffolds can promote cell proliferation and alignment, support long-term survival and maturation, and enhance function properties of cardiomyocytes.

\section{CONCLUSION}

Numerous reports provided compelling evidence that graphene can play an essential role in creating a more physiologically

\section{REFERENCES}

Ahadian, S., Zhou, Y., Yamada, S., Estili, M., Liang, X., Nakajima, K., et al. (2016). Graphene Induces Spontaneous Cardiac Differentiation in Embryoid Bodies. Nanoscale 8, 7075-7084. doi:10.1039/c5nr07059g

Akhavan, O., and Ghaderi, E. (2010). Toxicity of Graphene and Graphene Oxide Nanowalls against Bacteria. ACS Nano 4, 5731-5736. doi:10.1021/nn101390x

Ameri, S. K., Singh, P. K., D’angelo, R., Stoppel, W., Black, L., and Sonkusale, S. R. (2016). Three Dimensional Graphene Scaffold for Cardiac Tissue Engineering and Iin-Ssitu Electrical Recording. Annu. Int. Conf. IEEE Eng. Med. Biol. Soc. 2016, 4201-4203. doi:10.1109/EMBC.2016.7591653

Annabi, N., Shin, S. R., Tamayol, A., Miscuglio, M., Bakooshli, M. A., Assmann, A., et al. (2016). Highly Elastic and Conductive Human-Based Protein Hybrid Hydrogels. Adv. Mater. 28, 40-49. doi:10.1002/adma.201503255

Bahrami, S., Baheiraei, N., Mohseni, M., Razavi, M., Ghaderi, A., Azizi, B., et al. (2019). Three-dimensional Graphene Foam as a Conductive Scaffold for Cardiac Tissue Engineering. J. Biomater. Appl. 34, 74-85. doi:10.1177/0885328219839037

Barbolina, I., Woods, C. R., Lozano, N., Kostarelos, K., Novoselov, K. S., and Roberts, I. S. (2016). Purity of Graphene Oxide Determines its Antibacterial Activity. 2d Mater. 3, 025025. doi:10.1088/2053-1583/3/2/025025

Bolotin, K. I., Sikes, K. J., Jiang, Z., Klima, M., Fudenberg, G., Hone, J., et al. (2008). Ultrahigh Electron Mobility in Suspended Graphene. Solid State. Commun. 146, 351-355. doi:10.1016/j.ssc.2008.02.024

Brenner, D. W., Shenderova, O. A., Harrison, J. A., Stuart, S. J., Ni, B., and Sinnott, S. B. (2002). A Second-Generation Reactive Empirical Bond Order (REBO) accurate microenvironment for cardiac cells and EHTs. However, more research needs to be done to optimize the composition and geometry of graphene-based scaffolds for complex long-term in vivo applications. It is important to note that the graphene research is still in its infancy, and new fundamental discoveries and new practical applications are emerging every day, further fueling the development of the next wave of biomedical applications. We wholeheartedly share the sentiment expressed by scientists from the University of Manchester, a home of 2010 Nobel Prize Laureates for the research on graphene that "the potential of graphene is limited only by our imagination".

\section{AUTHOR CONTRIBUTIONS}

AS, DK, RY, IE, and EM contributed substantially to this manuscript. All authors approve the publication of this manuscript and agree to be accountable for the content of the work.

\section{FUNDING}

This work was supported by R21-HL152324 (AS, IE, and EM), 1R43TR003252-01A1 (AS), 1R43TR001911-01A1 (AS), 1R43MH124563-01A1 (AS), the American Heart Association (19PRE34380781; RY), the Leducq Foundation project RHYTHM and the National Institutes of Health (R01HL141470, IE). The study (AS) was partly funded through a National Institutes of Health and Food and Drug Administration U01 Grant (5 U01 FD006676-02) to the Health and Environmental Sciences Institute (HESI).

Potential Energy Expression for Hydrocarbons. J. Phys. Condens. Matter 14 783-802. doi:10.1088/0953-8984/14/4/312

Castro Neto, A. H., Guinea, F., Peres, N. M. R., Novoselov, K. S., and Geim, A. K. (2009). The Electronic Properties of Graphene. Rev. Mod. Phys. 81, 109-162. doi:10.1103/revmodphys.81.109

Chen, J.-H., Jang, C., Xiao, S., Ishigami, M., and Fuhrer, M. S. (2008). Intrinsic and Extrinsic Performance Limits of Graphene Devices on SiO2. Nat. Nanotech 3, 206-209. doi:10.1038/nnano.2008.58

Chen, X., Feng, B., Zhu, D.-Q., Chen, Y.-W., Ji, W., Ji, T.-J., et al. (2019) Characteristics and Toxicity Assessment of Electrospun Gelatin/PCL Nanofibrous Scaffold Loaded with Graphene In Vitro and In Vivo. Ijn 14, 3669-3678. doi:10.2147/ijn.s204971

Fakhrali, A., Nasari, M., Poursharifi, N., Semnani, D., Salehi, H., Ghane, M., et al. (2021). Biocompatible Graphene-embedded PCL / PGS -based Nanofibrous Scaffolds: A Potential Application for Cardiac Tissue Regeneration. J. Appl. Polym. Sci. 138, 51177. doi:10.1002/app.51177

Fernández-Merino, M. J., Guardia, L., Paredes, J. I., Villar-Rodil, S., SolísFernández, P., Martínez-Alonso, A., et al. (2010). Vitamin C Is an Ideal Substitute for Hydrazine in the Reduction of Graphene Oxide Suspensions. The J. Phys. Chem. C. 114, 6426-6432. doi:10.1021/jp100603h

Geim, A. K. (2009). Graphene: Status and Prospects. Science 324, 1530-1534. doi:10.1126/science.1158877

Geim, A., and Novoselov, K. (2007). The Rise of Graphene. Nat. Mater. 6, 183-191. doi:10.1038/nmat1849

Ghasemi, A., Imani, R., Yousefzadeh, M., Bonakdar, S., Solouk, A., and Fakhrzadeh, H. (2019). Studying the Potential Application of Electrospun Polyethylene 
Terephthalate/graphene Oxide Nanofibers as Electroconductive Cardiac Patch. Macromol. Mater. Eng. 304, 1900187. doi:10.1002/mame.201900187

Hitscherich, P., Aphale, A., Gordan, R., Whitaker, R., Singh, P., Xie, L.-H., et al. (2018). Electroactive Graphene Composite Scaffolds for Cardiac Tissue Engineering. J. Biomed. Mater. Res. 106, 2923-2933. doi:10.1002/jbm.a.36481

Hu, C., Xue, J., Dong, L., Jiang, Y., Wang, X., Qu, L., et al. (2016). Scalable Preparation of Multifunctional Fire-Retardant Ultralight Graphene Foams. ACS Nano 10, 1325-1332. doi:10.1021/acsnano.5b06710

Hu, W., Peng, C., Luo, W., Lv, M., Li, X., Li, D., et al. (2010). Graphene-based Antibacterial Paper. ACS Nano 4, 4317-4323. doi:10.1021/nn101097v

Jiang, L., Chen, D., Wang, Z., Zhang, Z., Xia, Y., Xue, H., et al. (2019). Preparation of an Electrically Conductive Graphene Oxide/chitosan Scaffold for Cardiac Tissue Engineering. Appl. Biochem. Biotechnol. 188, 952-964. doi:10.1007/ s12010-019-02967-6

Jing, X., Mi, H.-Y., Napiwocki, B. N., Peng, X.-F., and Turng, L.-S. (2017). Musselinspired Electroactive Chitosan/graphene Oxide Composite Hydrogel with Rapid Self-Healing and Recovery Behavior for Tissue Engineering. Carbon 125, 557-570. doi:10.1016/j.carbon.2017.09.071

Karimi Hajishoreh, N., Baheiraei, N., Naderi, N., and Salehnia, M. (2020). Reduced Graphene Oxide Facilitates Biocompatibility of Alginate for Cardiac Repair. J. Bioactive Compatible Polym. 35, 363-377. doi:10.1177/0883911520933913

Kenry, Lee, W. C., Loh, K. P., and Lim, C. T. (2018). When Stem Cells Meet Graphene: Opportunities and Challenges in Regenerative Medicine. Biomaterials 155, 236-250. doi:10.1016/j.biomaterials.2017.10.004

Khosroshahi, Z., Kharaziha, M., Karimzadeh, F., and Allafchian, A. (2018). Green Reduction of Graphene Oxide by Ascorbic Acid. AIP Conf. Proc. 1920, 020009. doi:10.1063/1.5018941

Kim, T., Kahng, Y. H., Lee, T., Lee, K., and Kim, D. H. (2013). Graphene Films Show Stable Cell Attachment and Biocompatibility with Electrogenic Primary Cardiac Cells. Mol. Cell 36, 577-582. doi:10.1007/s10059-013-0277-5

Krueger, E., Chang, A. N., Brown, D., Eixenberger, J., Brown, R., Rastegar, S., et al. (2016). Graphene Foam as a Three-Dimensional Platform for Myotube Growth. ACS Biomater. Sci. Eng. 2, 1234-1241. doi:10.1021/ acsbiomaterials.6b00139

Lasocka, I., Szulc-Dąbrowska, L., Skibniewski, M., Skibniewska, E., GregorczykZboroch, K., Pasternak, I., et al. (2021). Cytocompatibility of Graphene Monolayer and its Impact on Focal Cell Adhesion, Mitochondrial Morphology and Activity in BALB/3T3 Fibroblasts. Materials 14, 643. doi:10.3390/ma14030643

Lee, C., Wei, X., Kysar, J. W., and Hone, J. (2008). Measurement of the Elastic Properties and Intrinsic Strength of Monolayer Graphene. Science 321, 385-388. doi:10.1126/science.1157996

Lee, J., Manoharan, V., Cheung, L., Lee, S., Cha, B.-H., Newman, P., et al. (2019). Nanoparticle-based Hybrid Scaffolds for Deciphering the Role of Multimodal Cues in Cardiac Tissue Engineering. ACS nano 13, 12525-12539. doi:10.1021/ acsnano.9b03050

Lee, T.-J., Park, S., Bhang, S. H., Yoon, J.-K., Jo, I., Jeong, G.-J., et al. (2014). Graphene Enhances the Cardiomyogenic Differentiation of Human Embryonic Stem Cells. Biochem. Biophysical Res. Commun. 452, 174-180. doi:10.1016/ j.bbrc.2014.08.062

Lesiak, B., Trykowski, G., Tóth, J., Biniak, S., Kövér, L., Rangam, N., et al. (2021). Chemical and Structural Properties of Reduced Graphene Oxide-Dependence on the Reducing Agent. J. Mater. Sci. 56, 3738-3754. doi:10.1007/s10853-020-05461-1

Meric, I., Han, M. Y., Young, A. F., Ozyilmaz, B., Kim, P., and Shepard, K. L. (2008). Current Saturation in Zero-Bandgap, Top-Gated Graphene Field-Effect Transistors. Nat. Nanotech 3, 654-659. doi:10.1038/nnano.2008.268

Nazari, H., Azadi, S., Hatamie, S., Zomorrod, M. S., Ashtari, K., Soleimani, M., et al. (2019). Fabrication of Graphene-silver/polyurethane Nanofibrous Scaffolds for Cardiac Tissue Engineering. Polym. Adv. Technol. 30, 2086-2099. doi:10.1002/ pat.4641

Nazari, H., Heirani-Tabasi, A., Hajiabbas, M., Khalili, M., Shahsavari Alavijeh, M., Hatamie, S., et al. (2020). Incorporation of Two-dimensional Nanomaterials into Silk Fibroin Nanofibers for Cardiac Tissue Engineering. Polym. Adv. Technol. 31, 248-259. doi:10.1002/pat.4765

Norahan, M. H., Pourmokhtari, M., Saeb, M. R., Bakhshi, B., Soufi Zomorrod, M., and Baheiraei, N. (2019). Electroactive Cardiac Patch Containing Reduced Graphene Oxide with Potential Antibacterial Properties. Mater. Sci. Eng. C. 104, 109921. doi:10.1016/j.msec.2019.109921
Novoselov, K. S., Fal'ko, V. I., Colombo, L., Gellert, P. R., Schwab, M. G., and Kim, K. (2012). A Roadmap for Graphene. Nature 490, 192-200. doi:10.1038/nature11458

Novoselov, K. S., Geim, A. K., Morozov, S. V., Jiang, D., Katsnelson, M. I., Grigorieva, I. V., et al. (2005). Two-dimensional Gas of Massless Dirac Fermions in Graphene. Nature 438, 197-200. doi:10.1038/nature04233

Novoselov, K. S., Geim, A. K., Morozov, S. V., Jiang, D., Zhang, Y., Dubonos, S. V., et al. (2004). Electric Field Effect in Atomically Thin Carbon Films. Science 306, 666-669. doi:10.1126/science.1102896

Pang, L., Dai, C., Bi, L., Guo, Z., and Fan, J. (2017). Biosafety and Antibacterial Ability of Graphene and Graphene Oxide In Vitro and In Vivo. Nanoscale Res. Lett. 12, 564. doi:10.1186/s11671-017-2317-0

Park, J., Kim, B., Han, J., Oh, J., Park, S., Ryu, S., et al. (2015). Graphene Oxide Flakes as a Cellular Adhesive: Prevention of Reactive Oxygen Species Mediated Death of Implanted Cells for Cardiac Repair. ACS nano 9, 4987-4999. doi: $10.1021 / \mathrm{nn} 507149 \mathrm{w}$

Park, M. V. D. Z., Bleeker, E. A. J., Brand, W., Cassee, F. R., Van Elk, M., Gosens, I., et al. (2017). Considerations for Safe Innovation: The Case of Graphene. ACS Nano 11, 9574-9593. doi:10.1021/acsnano.7b04120

Paul, A., Hasan, A., Kindi, H. A., Gaharwar, A. K., Rao, V. T. S., Nikkhah, M., et al. (2014). Injectable Graphene Oxide/hydrogel-Based Angiogenic Gene Delivery System for Vasculogenesis and Cardiac Repair. ACS nano 8, 8050-8062. doi:10.1021/nn5020787

Pinto, A. M., Gonçalves, I. C., and Magalhães, F. D. (2013). Graphene-based Materials Biocompatibility: a Review. Colloids Surf. B: Biointerfaces 111, 188-202. doi:10.1016/j.colsurfb.2013.05.022

Rastogi, S. K., Bliley, J., Matino, L., Garg, R., Santoro, F., Feinberg, A. W., et al. (2020). Three-dimensional Fuzzy Graphene Ultra-microelectrodes for Subcellular Electrical Recordings. Nano Res. 13, 1444-1452. doi:10.1007/ s12274-020-2695-y

Rastogi, S. K., Raghavan, G., Yang, G., and Cohen-Karni, T. (2017). Effect of Graphene on Nonneuronal and Neuronal Cell Viability and Stress. Nano Lett. 17, 3297-3301. doi:10.1021/acs.nanolett.7b01215

Regis, J., Vargas, S., Irigoyen, A., Bramasco-Rivera, E., Bañuelos, J. L., Delfin, L. C., et al. (2021). Near-UV Light Assisted green Reduction of Graphene Oxide Films through L-Ascorbic Acid. Int. J. Smart Nano Mater. 12, 20-35. doi:10.1080/ 19475411.2021.1887396

Reina, G., González-Domínguez, J. M., Criado, A., Vázquez, E., Bianco, A., and Prato, M. (2017). Promises, Facts and Challenges for Graphene in Biomedical Applications. Chem. Soc. Rev. 46, 4400-4416. doi:10.1039/c7cs00363c

Ryan, A. J., Kearney, C. J., Shen, N., Khan, U., Kelly, A. G., Probst, C., et al. (2018). Electroconductive Biohybrid Collagen/pristine Graphene Composite Biomaterials with Enhanced Biological Activity. Adv. Mater. 30, 1706442. doi:10.1002/adma.201706442

Savchenko, A., Cherkas, V., Liu, C., Braun, G. B., Kleschevnikov, A., Miller, Y. I., et al. (2018). Graphene Biointerfaces for Optical Stimulation of Cells. Sci. Adv. 4, eaat0351. doi:10.1126/sciadv.aat0351

Seifi, T., and Kamali, A. R. (2021). Anti-pathogenic Activity of Graphene Nanomaterials: A Review. Colloids Surf. B: Biointerfaces 199, 111509. doi:10.1016/j.colsurfb.2020.111509

Shin, S. R., Aghaei-Ghareh-Bolagh, B., Gao, X., Nikkhah, M., Jung, S. M., Dolatshahi-Pirouz, A., et al. (2014). Layer-by-Layer Assembly of 3D Tissue Constructs with Functionalized Graphene. Adv. Funct. Mater. 24, 6136-6144. doi:10.1002/adfm. 201401300

Shin, S. R., Zihlmann, C., Akbari, M., Assawes, P., Cheung, L., Zhang, K., et al. (2016). Reduced Graphene Oxide-GelMA Hybrid Hydrogels as Scaffolds for Cardiac Tissue Engineering. Small 12, 3677-3689. doi:10.1002/ smll.201600178

Smith, A. S. T., Yoo, H., Yi, H., Ahn, E. H., Lee, J. H., Shao, G., et al. (2017). Micro- and Nano-Patterned Conductive Graphene-PEG Hybrid Scaffolds for Cardiac Tissue Engineering. Chem. Commun. 53, 7412-7415. doi:10.1039/c7cc01988b

Smith, A. T., Lachance, A. M., Zeng, S., Liu, B., and Sun, L. (2019). Synthesis, Properties, and Applications of Graphene Oxide/reduced Graphene Oxide and Their Nanocomposites. Nano Mater. Sci. 1, 31-47. doi:10.1016/j.nanoms.2019.02.004

Talebi, A., Labbaf, S., Karimzadeh, F., Masaeli, E., and Nasr Esfahani, M.-H. (2020). Electroconductive Graphene-Containing Polymeric Patch: a Promising Platform for Future Cardiac Repair. ACS Biomater. Sci. Eng. 6, 4214-4224. doi:10.1021/acsbiomaterials.0c00266 
Savchenko et al.

Graphene-Based Cardiac Scaffolds

Tielrooij, K. J., Song, J. C. W., Jensen, S. A., Centeno, A., Pesquera, A., Zurutuza Elorza, A., et al. (2013). Photoexcitation cascade and Multiple Hot-Carrier Generation in Grapheme. Nat. Phys 9, 248-252. doi:10.1038/nphys2564

Tui, J. H., Leonard, A., Camp, N. D., Long, J. T., Nawas, Z. Y., Chavanachat, R., et al. (2021). Tunable Electroconductive Decellularized Extracellular Matrix Hydrogels for Engineering Human Cardiac Microphysiological Systems. Biomaterials 272, 120764. doi:10.1016/j.biomaterials.2021.120764

Wang, J., Gui, C., Nan, H., Yo, Y., Xiao, Y., Poon, E., et al. (2017). Graphene SheetInduced Global Maturation of Cardiomyocytes Derived from Human Induced Pluripotent Stem Cells. ACS Apple. Mater. Inter. 9, 25929-25940. doi:10.1021/ acsami.7b08777

Wick, P., Louw-Gaume, A. E., Kucki, M., Krug, H. F., Kostarelos, K., Fadeel, B., et al. (2014). Classification Framework for Graphene-Based Materials. Anger. Chem. Int. Ed. 53, 7714-7718. doi:10.1002/anie.201403335

Chang, F., Zhang, N., Meng, H.-X., Lu, H.-X., Lu, Y.-Q., Liu, C.-M., et al. (2019). Easy Applied Gelatin-Based Hydrogel System for Long-Term Functional Cardiomyocyte Culture and Myocardium Formation. ACS Biomater. Sci. Eng. 5, 3022-3031. doi:10.1021/acsbiomaterials.9b00515

Chang, Y., Tan, Y.-W., Stormer, H. L., and Kim, P. (2005). Experimental Observation of the Quantum Hall Effect and Berry's Phase in Grapheme. Nature 438, 201-204. doi:10.1038/nature04235

Zhao, G., Wing, H., Huang, G., Genin, G. M., Lu, T. J., Luo, Z., et al. (2018). Reduced Graphene Oxide Functionalized Nanofibrous Silk Fibroin Matrices for Engineering Excitable Tissues. NPG Asia Mater. 10, 982-994. doi:10.1038/s41427-018-0092-8
Zhao, L. (2019). A Novel Graphene Oxide Polymer Gel Platform for Cardiac Tissue Engineering Application. 3 Biotech. 9, 401-411. doi:10.1007/s13205-019$1912-4$

Conflict of Interest: AS and EM are co-founders of Nanotools Bioscience, a startup company developing novel graphene-based bioapplications. IE is cofounder of Cardialen and NuSera Biosystems, which develop implantable and interventional cardiac devices. DK and RY declare that the research was conducted in the absence of any commercial or financial relationships that could be construed as a potential conflict of interest.

Publisher's Note: All claims expressed in this article are solely those of the authors and do not necessarily represent those of their affiliated organizations, or those of the publisher, the editors and the reviewers. Any product that may be evaluated in this article, or claim that may be made by its manufacturer, is not guaranteed or endorsed by the publisher.

Copyright (C) 2021 Savchenko, Yin, Kireev, Efimov and Molokanova. This is an openaccess article distributed under the terms of the Creative Commons Attribution License (CC BY). The use, distribution or reproduction in other forums is permitted, provided the original author (s) and the copyright owner (s) are credited and that the original publication in this journal is cited, in accordance with accepted academic practice. No use, distribution or reproduction is permitted which does not comply with these terms.

Frontiers in Bioengineering and Biotechnology | www.frontiersin.org

8

December 2021 | Volume 9 | Article 797340 